\title{
Comparative Analysis on Blood Fatigue Variables after Isokinetic and Isotonic Exercise Training in Elite Athletes
}

\author{
Seong-Wook Seo, PT, MS · Kyoung Kim, PT, $\mathrm{PhD}^{1 \dagger}$. Sang-Cheol Im, PT, PhD \\ Department of Rehabilitation Sciences, Graduate School, Daegu University \\ ${ }^{1}$ Department of Physical Therapy, College of Rehabilitation Sciences, Daegu University
}

Received: November 72021 / Revised: November 82021 / Accepted: December 292021

(C) 2022 J Korean Soc Phys Med

\author{
| Abstract | \\ PURPOSE: This study examined the changes in the blood \\ fatigue variables caused by isokinetic and isotonic exercise \\ training.
}

METHODS: Ten healthy adult males with at least one year of athletic experience participated. The participants performed the isokinetic circuit exercise program first, followed by an isotonic circuit exercise program. A two-hour break was allowed between the isokinetic circuit exercise program and the isotonic circuit exercise program. The circuit exercise program consisted of four items (Squat, Deadlift, Shoulder press, and Bench press). The blood samples were analyzed for the LDH, CPK, and Cortisol levels.

RESULTS: The LDH level in the isokinetic group was significantly different from the isotonic group. In particular, the change in LDH level in the isokinetic group was $33.30 \%$ lower than that of the isotonic group. The serum CPK level of the isokinetic group showed a $10.03 \%$ lower decrease than the isotonic group, but the difference was not significant. The Cortisol level was relatively unchanged in the isotonic group,

$\dagger$ Corresponding Author : Kyoung Kim

kykim257@hanmail.net, https://orcid.org/0000-0003-4169-6852 This is an Open Access article distributed under the terms of the Creative Commons Attribution Non-Commercial License (http://creativecommons.org/licenses/by-nc/3.0) which permits unrestricted non-commercial use, distribution, and reproduction in any medium, provided the original work is properly cited. but it decreased in the isokinetic group. On the other hand, the Cortisol level did not show a significant difference between the two groups.

CONCLUSION: The isokinetic group showed alleviation of the three indices, unlike the isotonic group. Further studies associated with the changes in blood fatigue variables through various exercise programs and exercise intensity will be needed.

Key Words: Blood fatigue variables, Circuit training, Isokinetic exercise training, Isotonic exercise training

\section{Introduction}

Physical imbalances are generated by excessive energy intake and insufficient physical activity that ultimately leads to serious health problems, such as heart disease, diabetes, cancer, stroke, chronic lung and kidney diseases, and Alzheimer's disease [1]. Various chronic diseases can be alleviated by engaging in an ongoing healthy lifestyle, including regular exercise and appropriate physical activities [2]. Accordingly, the interest in weight-training sports, including weightlifting, bodybuilding, powerlifting, strongman competition, and CrossFit, has increased annually [3]. On the other hand, overtraining or overuse due to overflowing information and incorrect skill acquisition 
can cause injury [4]. Malina reported that weight training relates to a large variety of dynamic resistance training based on progressive overload and improves muscular strength and endurance [5]. Resistance training plays a substantial role in improving the athletic performance by elevating muscular strength, hypertrophy, power and speed, local muscular endurance, balance, motor performance, and coordination [6]. In addition, resistance training helps maintain body composition (height and body weight) and bone mineral content and reduces sports injuries. Herein, resistance training (two or three times per week) results in meaningful improvement in muscular strength during childhood and early adolescence. Among these weight training studies, studies on two types of active resistance training for isokinetic and isotonic exercise training have been widely studied [5,7].

Power is the amount of work (force $\mathrm{x}$ distance) divided by time; it is influenced by both speed and strength. The power or peak can be measured using isotonic or isokinetic modes with a dynamometer [8]. Isokinetic exercise training, which is a form of dynamic exercise, predetermines and holds the constant velocity of the muscle (lengthening and shortening) and the angular limb velocity by a rate-limiting device known as isokinetic special dynamometer like Cybex, Biodex, and Kin-COM [9,10]. The resistance is equivalent in the case of the muscular forces imposed throughout the range of movement and provides the optimal load to the muscles. Hence, resistance has generally been used to measure the muscular forces in dynamic conditions [11]. Moreover, isokinetic exercise training has been successful in rehabilitating osteoarthritis, anterior cruciate ligament reconstruction, muscle weakness, jumping capacity in athletes, and Parkinson's disease [12]. Munhoz da Silveira Campos et al. recently reported that aerobic plus resistance training associated with a long-term weight loss program and isokinetic parameters could reduce body fat and improve muscle strength [13]. Isotonic exercise training has been used in clinical settings and sports fields to prevent the loss of muscle strength or sarcopenia, including the accelerated loss of muscle mass and function [14] and has been incorporated into early rehabilitation programs [12]. Accordingly, isotonic exercise training is an exercise that changes the length and speed of muscles while folding and unfolding the joints. Thus, isotonic exercise is a useful exercise to increase the size of the muscles [8]. Ultimately, exercise training is an exercise program that induces dynamic muscle contraction to improve strength and endurance. During rehabilitation exercise, the type of tissue damage or disease, the stage of tissue healing, the state of the joint, and the goal of the exercise program will determine which type of exercise to proceed with [15].

Circuit training combines several exercises targeting different muscles with only a short rest between 12-15 repetitions $[16,17]$. Circuit training can increase lower and upper body strength. Above all, it can be applied safely in metabolically challenged people who suffer from disorders, such as hypertension, chronic heart failure, obesity, borderline coronary heart disease, and non-insulindependent diabetes mellitus (NIDDM) [18].

Physical exercise affects the homeostasis of the internal environment of the body. Fatigue is a physical exerciseinduced inability to achieve the desired work output. Physical fatigue, including excessive physical loading, mental strain/pressure, inadequate rest, is accompanied by a decline in functional performance. Thus, repeated fatigue causes constant damage to the muscular apparatus that can lead to changes in serum constituent enzymes, such as lactate dehydrogenase (LDH) and creatine phosphokinase (CPK) [19]. These enzymes have been studied by many researchers before and after exercise and can be used to confirm muscle diseases [20,21]. Moreover, physical fatigue, which means a stress situation, can lead to the hypersecretion of serum Cortisol after physical exercise training [22]. These blood fatigue variables (LDH, CPK, and Cortisol) are considered the limiting factors of exercise capacity. 
Despite the many studies on different types of muscle contraction, few studies have examined the effects of isokinetic and isotonic exercise on the blood fatigue parameters. This study compared the changes in blood fatigue parameters after performing isokinetic and isotonic exercise training, which is being introduced to rehabilitation exercise therapy after an injury. These results are intended to provide basic data for setting the exercise design and direction when performing a rehabilitation exercise program. This study hypothesized that there would be differences in the blood fatigue parameters according to the isokinetic and isotonic exercise training methods.

\section{Methods}

\section{Participants}

Among the 12 male adults living in Daegu with more than one year of exercise experience, 10 people who do not correspond to the following exclusion criteria were selected. The inclusion criteria were not tested on isokinetic dynamometers or participated in exercise-related studies. The exclusion criteria were a history of respiratory and cardiovascular disease, musculoskeletal disease, or injury, including ankle in the past six months, and a history of neuromuscular disease in the past six months [8]. All participants signed a consent form after receiving detailed explanations on the purpose and method of this study, which was approved by the Daegu University Institutional Review Board (1040621-201907-HRBR-041-02), before commencing the study. G-power 3.1.9.4 was used to determine the sample size. The effect size of the static balance variable of a previous study that examined the effects of the circuit exercise program was used [16]. Ten subjects were selected according to a calculated effect size of 1.20 , a significance level of .05 , and a power of $80 \%$ [23].

\section{Experimental procedure}

A xim machine (Ronfic, Busan, Korea) was used to set the exercise intensity of the circuit training exercise. Xim machine enables isokinetic and isotonic exercise, and is an instrument that can measure the precise muscle strength and speed of muscle contraction. In addition, it is equipped with a function to check and save real-time exercise information on a computer. The one-repetition maximum (1RM) of four circuit training exercise programs (Squat, deadlift, Shoulder press, and Bench press) was measured. The participants exercised at the same intensity and frequency of $70 \% \mathrm{RM}$ during the isokinetic and isotonic circuit training exercises. Before applying for this training program, a preliminary training program was practiced to familiarize the subjects with the circuit training methods and prevent injuries. The pre-training program was repeated 10 times at an intensity of $40-50 \%$ of $1 \mathrm{RM}$. This training program was applied 11 times at a 70\% intensity of 1RM using the xim machine computer measurement method (Table 1). After a two-hour break, the participants performed isokinetic circuit training first, followed by isotonic circuit training.

\section{Measurement methods}

The participants fasted for 12 hours after dinner the day before the experiment. Blood sampling was performed 30 minutes before and immediately after exercise in isokinetic and isotonic circuit training. The exercise program of this study was conducted at the hospital's health promotion center, and a professional nurse working in this hospital collected the blood samples. The participants collected $10 \mathrm{ml}$ of blood from the antecubital vein. The collected blood was centrifuged at 3,000 rpm for 10 minutes using a centrifuge (HA-1000-3, Hanil Industrial, Korea) to obtain a serum sample. After centrifugation, the supernatant was separated and analyzed by EONE Life Science Institute (Incheon, Korea). The serum LDH was measured using a SICDIA LDH reagent kit (Eiken Chemical, Tokyo, Japan) according to the manufacturer's protocols with a Hitachi 7600-110 (Hitachi, Tokyo, Japan). 
Table 1. Circuit Training Program

\begin{tabular}{|c|c|c|c|}
\hline \multicolumn{4}{|r|}{ Exercise Program } \\
\hline \multicolumn{3}{|c|}{ Warm um Exercise (10min) } & Stretching \\
\hline \multirow{4}{*}{$\begin{array}{l}\text { Circuit } \\
\text { training }\end{array}$} & Procedure 1 & Squat & $\begin{array}{l}\text { After holding the barbell behind the shoulders, stand with the legs shoulder-width } \\
\text { apart and toes pointing } 15^{\circ} \text {. Sit using only the lower body without bending the upper } \\
\text { body. Sit deeply so that the knee angle is } 90^{\circ} \text {, and return to the original position. }\end{array}$ \\
\hline & Procedure 2 & Deadlift & $\begin{array}{l}\text { From a sitting position with the legs shoulder-width apart, fix the waist like a bow, } \\
\text { and get up as if a barbell rubs against the legs. }\end{array}$ \\
\hline & Procedure 3 & Shoulder press & $\begin{array}{l}\text { Standing or sitting, grab a barbell that is twice shoulder-width apart and feet } \\
\text { shoulder-width apart. Raise the barbell above the chest. Lift the barbell over the head } \\
\text { from the chest. While feeling resistance in the shoulders, lower the bar until it is } \\
\text { level with the ears. }\end{array}$ \\
\hline & Procedure 4 & Bench press & $\begin{array}{l}\text { Lie on a bench and place the hips and shoulder blades on the backrest, arching the } \\
\text { back approximately } 10 \mathrm{~cm} \text {. Hold the bar twice shoulder-width apart and position } \\
\text { the eyes perpendicular to the barbell. Lift the bar and place it so that the center } \\
\text { of the chest and the bar are perpendicular, then bend the elbow slightly to fix it. } \\
\text { Push up on the barbell as you feel it tightening the armpits. }\end{array}$ \\
\hline \multicolumn{3}{|c|}{ Cooldown Exercise (5min) } & Stretching \\
\hline
\end{tabular}

The serum CPK activity was assayed using a commercial kit (L-Type CK; Wako Pure Chemical Industries, Osaka, Japan) with Hitachi 7600-110 (Hitachi, Tokyo, Japan). The serum cortisol level was determined by the electrochemiluminescence immunoassay (ECLIA) and measured using a Cortisol assay (Roche Diagnostics, Germany) on a Roche Cobas analyzer. The LDH is an enzyme that catalyzes the reaction when synthesizing ATP. The LDH concentration increases when the lactic acid produced by the muscles is released into the blood. CPK regulates the ATP levels during muscle contraction and is used as an energy source. Cortisol is responsible for storing the energy required by the body and making it easier to use energy sources when needed.

\section{Statistical analysis}

This study used SPSS version 25.0 software (SPSS Inc., Chicago, IL, USA) to analyze the data. The data are presented as the mean \pm standard deviation. The normality of the data was tested using a Shapiro-Wilk test. An independent t-test was used to compare the results of the two groups, and a paired t-test was used to compare the
Table 2. Participants Characteristics

$(n=10)$

\begin{tabular}{cc}
\hline Variable & Mean \pm SD \\
\hline Gender $(\mathrm{M} / \mathrm{F})$ & $10 / 0$ \\
Age (years) & $30.50 \pm 4.35$ \\
Height $(\mathrm{cm})$ & $178.10 \pm 4.20$ \\
Weight $(\mathrm{kg})$ & $79.31 \pm 11.17$ \\
Muscle $(\mathrm{kg})$ & $38.16 \pm 6.00$ \\
BMI $(\mathrm{kg} / \mathrm{m} 2)$ & $15.20 \pm 6.32$ \\
\hline
\end{tabular}

effects between the pre-and post-exercise after invention. $\mathrm{P}<.05$ was considered to indicate a significant difference.

\section{Results}

\section{General Characteristics of the participants}

Table 2 lists the general characteristics of the study participants.

\section{Change in serum LDH}

The LDH activity (IU/L) in the isokinetic group showed a significant difference between the pre-exercise and 


\begin{tabular}{|c|c|c|c|c|c|}
\hline \multirow{2}{*}{ Group } & \multicolumn{3}{|c|}{ LDH Level } & \multirow{2}{*}{$\mathrm{t}$} & \multirow{2}{*}{$\mathrm{p}$} \\
\hline & Pre & Post & Post-Pre & & \\
\hline Isokinetic exercise & $342.31 \pm 78.70$ & $439.37 \pm 87.25$ & $97.03 \pm 54.61$ & -5.027 & $.003^{* *}$ \\
\hline Isotonic exercise & $365.68 \pm 119.03$ & $511.05 \pm 138.44$ & $145.42 \pm 165.67$ & -2.324 & .059 \\
\hline $\mathrm{t}$ & -.454 & & -.739 & & \\
\hline $\mathrm{P}$ & .658 & & .483 & & \\
\hline
\end{tabular}

Mean $\pm \mathrm{SD}, \quad{ }^{* *} \mathrm{p}<.01$

Table 4. Change of CPK Level

\begin{tabular}{|c|c|c|c|c|c|}
\hline \multirow{2}{*}{ Group } & \multicolumn{3}{|c|}{ CPK level } & \multirow{2}{*}{$\mathrm{t}$} & \multirow{2}{*}{$\mathrm{p}$} \\
\hline & Pre & Post & Post-Pre & & \\
\hline Isokinetic exercise & $298.90 \pm 194.37$ & $330.32 \pm 208.25$ & $31.41 \pm 18.16$ & -5.499 & $<.001^{* * *}$ \\
\hline Isotonic exercise & $324.06 \pm 210.41$ & $358.92 \pm 223.44$ & $34.93 \pm 18.24$ & -6.052 & $<.001^{* * *}$ \\
\hline $\mathrm{t}$ & -.277 & & -.431 & & \\
\hline $\mathrm{p}$ & .785 & & .671 & & \\
\hline
\end{tabular}

Mean $\pm \mathrm{SD},{ }^{* * *} \mathrm{p}<.001$

post-exercise (342.31 \pm 78.70 and $439.37 \pm 87.25$, respectively). By contrast, the LDH activity in the isotonic group did not show a significant difference between pre-exercise and post-exercise (365.68 \pm 119.03 and $511.05 \pm 138.44$, respectively). The decrease in the LDH activity was $33.30 \%$ lower in the isokinetic group than in the isotonic group, but the difference was not significant (Table 3).

\section{Change in serum CPK}

The CPK activity (IU/L) in the isokinetic group was $298.90 \pm 194.37$ and $330.32 \pm 208.25$ in the pre-exercise and post-exercise groups, respectively. By contrast, the CPK activity in the isotonic group was $324.06 \pm 210.41$ and $358.92 \pm 223.44$ pre-exercise and post-exercise, respectively. The change in the CPK activity was $10.03 \%$ lower in the isokinetic group, but the difference was not significant (Table 4).

\section{Change of serum Cortisol}

The Cortisol level $(\mu \mathrm{g} / \mathrm{dL})$ in the isokinetic group was
$11.05 \pm 2.22$ and $10.26 \pm 2.20$ pre-exercise and post-exercise, respectively. By contrast, the Cortisol level in the isotonic group was $8.80 \pm 2.33$ and $8.87 \pm 2.61$ pre-exercise and post-exercise, respectively. The Cortisol level in the isokinetic group decreased after exercise, but the Cortisol level in the isotonic group increased after exercise (Table 5). There was no significance between the two groups.

\section{Discussion}

Despite the many studies on different types of muscle contractions, few studies have examined the effects of isokinetic and isotonic exercise on the blood fatigue parameters. Furthermore, studies on the effectiveness of isokinetic and isotonic exercise training report conflicting results [24]. Therefore, the present study compared and analyzed the effects of isokinetic versus isotonic exercise training in terms of blood fatigue variables, including $\mathrm{LDH}$, CPK, and Cortisol. The circuit exercise program used in this experiment consisted of four items (Squat, Dead Lift, 
Table 5. Change of Cortisol Level

\begin{tabular}{|c|c|c|c|c|c|}
\hline \multirow{2}{*}{ Group } & \multicolumn{3}{|c|}{ Cortisol level } & \multirow{2}{*}{$\mathrm{t}$} & \multirow{2}{*}{$\mathrm{p}$} \\
\hline & Pre & Post & Post-Pre & & \\
\hline Isokinetic exercise & $11.05 \pm 2.22$ & $10.26 \pm 2.20$ & $-.85 \pm 1.13$ & 2.042 & .075 \\
\hline Isotonic exercise & $8.80 \pm 2.33$ & $8.87 \pm 2.61$ & $.11 \pm 1.30$ & -.243 & .813 \\
\hline $\mathrm{t}$ & 1.960 & & -1.825 & & \\
\hline $\mathrm{p}$ & .072 & & .086 & & \\
\hline
\end{tabular}

Mean \pm SD

Shoulder Press, and Bench Press). The squat is considered a fundamental movement in athletic training and is used as an effective exercise commonly applied during patellofemoral or cruciate ligament rehabilitation [25]. Dead Lift exercise with barbells, dumbbells, and kettlebells activates all the muscles of the lower body, such as the legs, hips, back, and core muscles [26]. Shoulder Press has several benefits, including the development of strength and size of triceps muscles, trapezius muscle, and core muscles [27]. Moreover, the main three muscles like the pectoralis major, the anterior deltoid muscles, and the triceps brachii, develop when the bench press does [26].

Before and after isokinetic and isotonic exercise training, the blood fatigue variables, such as LDH, CPK, and Cortisol, were evaluated through the collected serum. Lactate dehydrogenase (LDH) is normally present at low concentrations. On the other hand, during high-intensity exercise, intracellular LDH is released from the cell due to muscle damage, increasing the $\mathrm{LDH}$ level. This increase in LDH level was evaluated as an indirect marker of muscle damage, and as an indicator of the exercise capacity and fatigue patterns based on energy metabolism [28]. Creatinine phosphokinase (CPK) was observed in most forms of strenuous exercise, approximately 20 times the normal range (usually 10 times the normal range when exercise-induced rhabdomyolysis) [29,30]. Buonocore D et al. (2020) suggested that physical exercise training leads to increased LDH and CPK. According to the above result, $\mathrm{LDH}$ and $\mathrm{CPK}$ in the two groups increased after exercise
[31]. The significance in the LDH level between post- and pre-exercise was observed only in the isokinetic group ( $p$ $<.01)$. The change in LDH level in the isokinetic group was $33.30 \%$ lower than that of the isotonic group. In addition, the serum CPK level of the isokinetic group showed a $10.03 \%$ lower decrease than the isotonic group, but the differences were not significant. The rest time between the exercise sets and recovery time after exercise can be affected by the exercise intensity, muscle activation (number and size of muscles mobilized), and microtrauma caused by stimulation (amount of muscle protein destroyed during exercise) [32]. In this experiment, isokinetic and isotonic exercise did not induce high fatigue. In addition, although the initial value of the isokinetic group was slightly higher than that of the isotonic group, it is not expected to affect the interpretation of the results because there was no significance when the independent t-test was performed. These results suggest that isokinetic exercise training is more beneficial for fatigue and recovery after exercise. A previous study reported that the isotonic exercise protocol caused a significant functional defect because of the decreased muscle strength after exercise more than the isotonic exercise protocol [33].

Cortisol, which plays a substantial role in regulating energy metabolism and homeostasis on the skeletal muscle, is regulated at the tissue and systemic level to maintain glucocorticoid homeostasis [34]. The cortisol level caused by exercise is highest when the overall stress of the training period increases [35]. The change in Cortisol between post- 
and pre-exercise was $-.85 \pm 1.13$ (at isokinetic group) and $.11 \pm 1.30$ (at isotonic group). The Cortisol level in the isokinetic group decreased after exercise, but the Cortisol level in the isotonic group increased after exercise. This result is consistent with previous studies reporting that intense resistance exercise increased the amount of cortisol and testosterone, but resistance exercise with low muscle use had little effect [36]. These blood fatigue parameters reflect muscle damage and are useful indicators for determining the condition of muscle tissue [37]. Overall, these results suggest that isokinetic circuit training exercise has partial advantages over isotonic circuit training exercise in muscle fatigue.

This study had some limitations. The sample size was relatively small, so generalization is limited. In addition, the participants were recruited only from men from Daegu area. In future experiments, more scientific results can be obtained by expanding the range of participants related to residence and gender. In addition, another limitation of this experiment is that it did not consider the factors that could affect the blood fatigue variables when they had underlying diseases as the participants were normal elite athletes.

\section{Conclusion}

The increase in the blood fatigue variable LDH was significantly smaller in isokinetic circuit training exercise than in the isotonic circuit training exercise. Thus, the isokinetic group reduced the blood fatigue variables more effectively than the isotonic group. Both isokinetic exercise and isotonic exercise have advantages and disadvantages. On the other hand, isokinetic exercise was evaluated to be more effective than isotonic exercise only when blood fatigue parameters were compared in this experiment. Nevertheless, more study is needed because there are various factors affecting the body.

\section{References}

[1] Hathaway E. Chronic disease prevention in faith-based organizations. J Pastoral Care Counsel. 2018;72(3): 159-62.

[2] Williams DM. Exercise, affect, and adherence: an integrated model and a case for self-paced exercise. J Sport Exerc Psychol. 2008;30(5):471-96.

[3] Keogh JW, Winwood PW. The epidemiology of injuries across the weight-training sports. Sports Med. 2017;47(3): 479-501.

[4] Siewe J, Rudat J, Röllinghoff M, et al. Injuries and overuse syndromes in powerlifting. Int J Sports Med. 2011;32(9): 703-11.

[5] Malina RM. Weight training in youth-growth, maturation, and safety: an evidence-based review. Clin J Sport Med. 2006;16(6):478-87.

[6] Kraemer WJ, Ratamess NA. Fundamentals of resistance training: progression and exercise prescription. Med Sci Sports Exerc. 2004;36(4):674-88.

[7] Schmitz RJ, Westwood KC. Knee extensor electromyographic activity-to-work ratio is greater with isotonic than isokinetic contractions. J Athl Train. 2001;36(4):384-7.

[8] Webber SC, Porter MM. Reliability of ankle isometric, isotonic, and isokinetic strength and power testing in older women. Phys Ther. 2010;90(8):1165-75.

[9] Lee SEK, Lira CAB, Nouailhetas VLA, et al. Do isometric, isotonic and/or isokinetic strength trainings produce different strength outcomes?. J Body Mov Ther. 2018; 22(2):430-7.

[10] Chen CL, Chang KJ, Wu PY, et al. Comparison of the effects between isokinetic and isotonic strength training in subacute stroke patients. J Stroke Cerebrovasc Dis. 2015;24(6):1317-23.

[11] Baltzopoulos V, Brodie DA. Isokinetic dynamometry. Applications and limitations. Sports Med. 1989;8(2): 101-16.

[12] Dauty M, Menu P, Fouasson-Chailloux A, et al. Muscular 
isokinetic strength recovery after knee anterior cruciate ligament reconstruction revision: preliminary study. Ann Phys Rehabil Med. 2014;57(1):55-65.

[13] Munhoz da Silveira Campos R, Moreira CE, Leão da Silva $\mathrm{P}$, et al. The effect of aerobic plus resistance training associated with a long-term interdisciplinary weight loss program on visceral fat and isokinetic parameters in adolescents with obesity. J Sports Med Phys Fitness. 2020;60(6):855-63.

[14] Cruz-Jentoft AJ, Sayer AA. Sarcopenia. Lancet. 2019; 393(10191):2636-46.

[15] Kim JS, Kim SS, Jang KM. The effects of different exercise type for maximal exercise on RPP and blood lactate. J Korean Acad Phys Ther. 2003;10(1):149-57.

[16] Jung WS, Kim YY, Park HY. Circuit training improvements in Korean women with sarcopenia. Percept Mot Skills. 2019;126(5):828-42.

[17] Buch A, Kis O, Carmeli E, et al. Circuit resistance training is an effective means to enhance muscle strength in older and middle aged adults: A systematic review and meta-analysis. Ageing Res Rev. 2017;37:16-27.

[18] Romero-Arenas S, Martínez-Pascual M, Alcaraz PE. Impact of resistance circuit training on neuromuscular, cardiorespiratory and body composition adaptations in the elderly. Aging Dis. 2013;4(5):256-63.

[19] Overgaard K, Fredsted A, Hyldal A, et al. Effects of running distance and training on $\mathrm{Ca} 2+$ content and damage in human muscle. Med Sci Sports Exerc. 2004;36(5): 821-9.

[20] Assenza A, Marafioti S, Congiu F, et al. Serum musclederived enzymes response during show jumping competition in horse. Vet World. 2016;9(3):251-5.

[21] Ament W, Verkerke GJ. Exercise and fatigue. Sports Med. 2009;39(5):389-422.

[22] Beserra AHN, Kameda P, Deslandes AC, et al. Can physical exercise modulate cortisol level in subjects with depression? A systematic review and meta-analysis. Trends Psychiatry Psychother. 2018;40(4):360-8.
[23] Faul F, Erdfelder E, Buchner A, et al. Statistical power analyses using G*Power 3.1: tests for correlation and regression analyses. Behav Res Methods 2009;41(4): 1149-60.

[24] Chen CL, Chang KJ, Wu PY, et al. Comparison of the effects between isokinetic and isotonic strength training in subacute stroke patients. J Stroke Cerebrovasc Dis. 2015;24(6):1317-23.

[25] Ryanton MA, Carey JP, Kennedy MD, et al. Quadriceps effort during squat exercise depends on hip extensor muscle strategy. Sports Biomech. 2015;14(1):122-38.

[26] Bengtsson V, Berglund L, Aasa U. Narrative review of injuries in powerlifting with special reference to their association to the squat, bench press and deadlift. BMJ Open Sport Exerc Med. 2018;4(1):e000382.

[27] Campos YAC, Vianna JM, Guimarães MP, et al. Different shoulder exercises affect the activation of deltoid portions in resistance-trained individuals. J Hum Kinet. 2020; 75:5-14.

[28] Philippou A, Maridaki M, Tenta R, et al. Hormonal responses following eccentric exercise in humans. Hormones. 2017;16(4):405-13.

[29] Lee G. Exercise-induced rhabdomyolysis. R I Med J. 2014;97(11):22-4.

[30] Meyer M, Sundaram S, Schafhalter-Zoppoth I. Exertional and crossfit-induced rhabdomyolysis. Clin J Sport Med. 2018;28(6):e92-4.

[31] Buonocore D, Verri M, Giolitto A, et al. Effect of 8-week n-3 fatty-acid supplementation on oxidative stress and inflammation in middle- and long-distance running athletes: a pilot study. J Int Soc Sports Nutr. 2020;17(1):55.

[32] Choi BS, Han SW. Effects of Resting Periods Between Exercise Sets During Isokinetic Exercise on Muscle Performances and Physiological Variables in Middle-aged Women. Phys Ther Korea. 2001;8(3):77-95.

[33] Parr JJ, Yarrow JF, Garbo CM, et al. Symptomatic and functional responses to concentric-eccentric isokinetic versus eccentric-only isotonic exercise. J Athl Train. 
2009;44(5):462-8.

[34] Munck A, Guyre PM, Holbrook NJ. Physiological functions of glucocorticoids in stress and their relation to pharmacological actions. Endocr Rev. 1984;5(1):25-44.

[35] Kraemer WJ, Ratamess NA, Hymer WC, et al. Growth hormone(s), testosterone, insulin-like growth factors, and cortisol: roles and integration for cellular development and growth with exercise. Front Endocrinol. 2020;11:33.
[36] Geisler S, Aussieker T, Paldauf S, et al. Salivary testosterone and cortisol concentrations after two different resistance training exercises. J Sports Med Phys Fitness. 2019;59(6):1030-5.

[37] Brancaccio P, Maffulli N, Buonauro R, et al. Serum enzyme monitoring in sports medicine. Clin Sports Med. 2008;27(1):1-18 